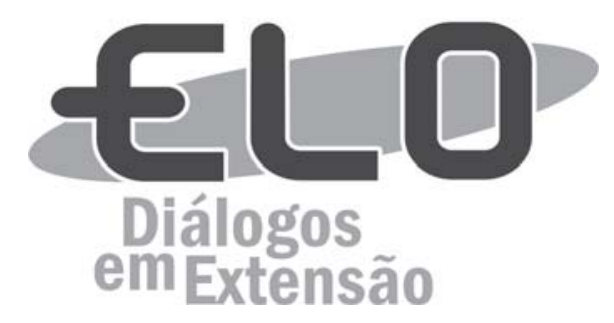

\title{
Ações em prol do aleitamento materno e doação de leite humano de um projeto de extensão universitária
}

\author{
Giulia Gonçalves Pena ${ }^{1}$, Patrícia Feliciano Pereira ${ }^{2}$, Tatiane Cristina \\ Serafim ${ }^{3}$, Raquel Maria Amaral Araújo ${ }^{4}$, Rafaela Mara Silva Fonseca ${ }^{5}$
}

\begin{abstract}
Resumo: O objetivo desse relato é descrever as ações do projeto de extensão "Incentivo ao aleitamento materno e doação de leite humano no município de Viçosa-MG" e discuti-las como promotoras de saúde materno-infantil. As atividades incluem a realização de campanhas de apoio ao aleitamento materno e divulgação do Banco de Leite Humano (BLH), realização de coleta domiciliar de leite materno, captação de doadoras e realização de orientações sobre amamentação. Comparando os anos de desenvolvimento do projeto (2015 e 2016) com o ano anterior, observou-se aumento no número de visitas domiciliares, no volume de leite coletado, no número de doadoras e de atendimentos individuais no BLH. Os resultados representam ganhos em termos de maior disponibilidade de leite humano destinado aos prematuros e número maior de mulheres apoiadas no processo da amamentação. Conclui-se que a Extensão Universitária contribui para potencializar ações de saúde pública em prol do aleitamento materno e da doação do leite humano.
\end{abstract}

Palavras-chave: Bancos de Leite. Aleitamento materno. Leite humano.

Área Temática: Educação e Saúde.

\section{Actions for Breastfeeding and donation human milk of an university extension project}

Abstract: The objective of this report is to describe the actions of the extension project "Incentive to breastfeeding and donation of human milk in the municipality of Viçosa-MG" and to discuss them as promoters of maternal and child health. Activities include campaigns to support breastfeeding and dissemination of the Human Milk Bank (HMB), home collection of breast milk, donor recruitment and provision of guidelines on breastfeeding. Comparing the project's years of development (2015 and 2016) with the previous year, there was an increase in the number of home visits, the volume of milk collected, the number of donors and individual visits in the HMB. The results represent gains in terms of greater availability of human milk for preterm infants and the greater number of women supported in the breastfeeding process. It is concluded that the University Extension contributes to the strengthening of public health actions in favor of breastfeeding and the donation of human milk

Keywords: Milk banks. Breastfeeding. Human milk.

\section{Acciones en favor de la lactancia materna y donación de leche humanan de un proyecto de extensión universitaria}

Resumen: El objetivo de este relato es describir las acciones del proyecto de extensión "Incentivo a la lactancia materna y donación de leche humana en el municipio de Viçosa-MG" y discutirlas como

${ }^{1}$ Graduada em Nutrição pela Universidade Federal de Viçosa; Bolsista PIBEX 2016.

${ }^{2}$ Professora Adjunta do Departamento de Nutrição e Saúde da Universidade Federal de Viçosa; Coordenadora do projeto de extensão. E-mail: patricia.feliciano@ufv.br Rua da Mantiqueira n.95, apt 201, João Brás, 36576-216, Viçosa-MG, (31) 983008282.

${ }^{3}$ Nutricionista do Departamento de Nutrição e Saúde da Universidade Federal de Viçosa.

${ }^{4}$ Professora Associada do Departamento de Nutrição e Saúde da Universidade Federal de Viçosa.

${ }^{5}$ Nutricionista do Banco de Leite Humano do Hospital São Sebastião, Viçosa-MG. 
promotoras de salud materna-infantil. Las actividades incluyen la realización de campañas de apoyo a la lactancia materna y divulgación del Banco de Leche Humana (BLH), realización de recolección domiciliar de leche materna, captación de donantes y suministro de orientaciones sobre lactancia. Comparando los resultados del desarrollo del proyecto (2015 y 2016) con respecto al anterior, se observó un aumento en el número de visitas domiciliares, ni el volumen de leche recogido, ni el número de donantes y en la assistência individual en el BLH. Los resultados representan ganancias en términos de mayor disponibilidad de leche humana destinada a los prematuros y mayor número de mujeres apoyadas en el proceso de la lactancia. Se concluye que la Extensión Universitaria contribuye a potenciar acciones de salud pública en favor de la lactancia materna y de la donación de la leche humana.

Palabras clave: Bancos de leche. Lactancia materna. Leche humana.

\section{Introdução}

O leite materno é um alimento completo para o lactente, pois contém quantidade adequada de proteínas, gorduras, vitaminas, minerais e imunoglobulinas que favorecem o crescimento e desenvolvimento adequado da criança. Considerando as suas propriedades benéficas, a Organização Mundial da Saúde (WHO, 2003) e o Ministério da Saúde recomendam o aleitamento materno exclusivo até os seis meses de idade, e de forma complementar, até os dois anos ou mais.

A maioria das mães sonha com o momento do nascimento do seu filho e idealizam o momento da amamentação, porém, muitas vezes elas são surpreendidas pela prematuridade do parto que, por sua vez, pode acarretar em dificuldades e até mesmo a impossibilidade da prática do aleitamento materno. Sabe-se que o leite materno é considerado o melhor alimento para o prematuro, pois possui nutrientes específicos que contribuem para maturação biológica, diminuem a incidência de infecções e favorecem o desenvolvimento cerebral desses bebês (SUCENA, 2008; QUIGLEY et al, 2018; TOSH, 2018). Quando o leite materno não está disponível ou está em falta, o leite doado aos Bancos de Leite Humano representa a segunda melhor alternativa alimentar para o prematuro (BERTINO et al, 2013; DeMARCHIS et al, 2017).

O Banco de Leite Humano (BLH) constitui-se neste cenário como importante estratégia da política pública em prol da amamentação, uma vez que desenvolve ações de apoio ao aleitamento materno e incentivo à doação do leite humano (BRASIL, 2015). O Brasil desenvolveu uma das mais complexas e eficientes redes de bancos de leite humano do mundo, construindo um instrumento nacional a fim de enfrentar o desafio da saúde na primeira infância (PRITTAS e DRI, 2017).

Criado em 2005 o BLH de Viçosa, MG, situa-se no Hospital São Sebastião (HSS), sendo considerado um importante aliado para a promoção da amamentação e doação de leite materno. O leite doado é destinado, principalmente, aos recém-nascidos prematuros internados na Unidade de Terapia Intensiva Neonatal (UTIN) (CASA DE CARIDADE DE VIÇOSA, 2013). Neste local, as mães são acolhidas, recebem orientações relacionadas à amamentação, realizam procedimentos para ordenha de alívio assim como para estímulo da produção láctea, trocam experiências com outras mães, esclarecem as dúvidas e, aquelas que podem e desejam, realizam a doação de leite materno (CORINTIO, 2015; PEREIRA et al, 2018).

O presente trabalho buscou descrever as ações realizadas no projeto de extensão "Incentivo ao aleitamento materno e doação de leite humano no município de Viçosa-MG" e discuti-las como instrumentos promotores da saúde materno-infantil.

\section{Metodologia}

O projeto de extensão: “Incentivo ao aleitamento materno e doação de leite humano no município de Viçosa-MG" teve início em fevereiro de 2015, como parceria entre o Departamento de Nutrição e Saúde da Universidade Federal de Viçosa e a Casa de Caridade Hospital São Sebastião (HSS). As ações desenvolvidas acontecem de forma integrada e constituem em desenvolver práticas de promoção e incentivo ao aleitamento materno, organizar e promover campanhas de doação de leite humano e divulgação do BLH; captar doadoras de leite humano; realizar coleta domiciliar de leite humano e realizar orientações às mães doadoras sobre amamentação e nutrição.

As campanhas são importantes para promoção da prática do aleitamento materno, divulgação da existência e funções do Banco de Leite Humano e como forma de captar nutrizes para serem 
doadoras de leite materno. Para a elaboração das mesmas são utilizados recursos diversos, incluindo divulgação de mensagens educativas a respeito da amamentação e doação de leite materno por meio de redes sociais, entrega de panfletos, folders e informativos às mães que vão ao BLH ou no momento da coleta domiciliar. Para atingir a comunidade em geral são feitas campanhas temáticas em praças públicas ou até mesmo na maternidade do HSS. Entre estas, destacam-se o Dia Mundial de Doação de Leite Humano comemorado no dia 19 de maio, Dia nacional de Doação de Leite Humano comemorado no dia $1^{\circ}$ de outubro, a Semana Mundial de Aleitamento Materno que acontece na semana do dia 1 a 7 de agosto, além de campanhas para doação de frascos para armazenamento do leite, e o Encontro Anual de Doadoras de Leite do BLH que é realizado no mês de dezembro no próprio Hospital.

A captação de doadoras acontece também por meio de ligações telefônicas às mães cujos contatos são obtidos no caderno de anotações de partos ocorridos no Hospital São Sebastião. As ligações são feitas por volta de 15 dias a um mês pós-parto para orientar e sanar possíveis dúvidas acerca da amamentação, além de saber, o possível interesse e possibilidade de se tornarem doadoras.

Uma das principais propostas do projeto é a coleta domiciliar de leite materno. Semanalmente são feitas ligações telefônicas às nutrizes cadastradas, a fim de saber se as mesmas desejam fazer alguma doação e orientá-las quanto aos cuidados de higiene na ordenha para preservar a qualidade do leite. Aquelas que possuem leite para ser doado são recebidas no domicilio por um estudante de nutrição, previamente treinado, para a realização da coleta. O leite doado, já congelado, é transportado em um carro da UFV, segundo as normas da Agência Nacional de Vigilância Sanitária (BRASIL, 2008).

Após o recebimento, os frascos são higienizados através da aplicação de álcool $70 \%$ na parte externa, friccionando-o por 15 segundos, tendo cuidado de lavar em água corrente os frascos contendo camada externa de gelo. Verifica-se então a adequação do preenchimento do rótulo da embalagem na qual o leite está acondicionado e as condições de conservação na qual o leite se encontra no momento da recepção. Em seguida, registra-se possíveis alterações nos frascos, bem como a presença de sujidades no leite, desprezando-os se for o caso. O volume e identificação do leite doado para que haja rastreamento do mesmo, é registrado em banco de dados do BLH e posteriormente lançados no site da Rede Global de Banco de Leite Humano da Fiocruz. Após a seleção e classificação, os frascos são encaminhados para o teste de Crematócrito, é feito o reenvase, pasteurização, resfriamento e posteriormente a análise microbiológica. Após o processamento, o leite biologicamente seguro está adequado ao consumo e pode ser mantido em freezer no prazo máximo de 6 meses à temperatura de $-10^{\circ} \mathrm{C}$ ou inferior a esta.

O acompanhamento das doadoras é feito em cada visita domiciliar, momento no qual a nutriz é questionada sobre intercorrências mamárias e alimentação saudável. Caso haja identificação de qualquer necessidade, a mãe é encaminhada para atendimento médico e ou nutricional no Hospital São Sebastião.

Para quantificar o impacto de todas as atividades desenvolvidas foram comparados os indicadores antes e após o início do projeto. Os indicadores avaliados foram obtidos no site da Rede Global de Banco de Leite Humano da Fiocruz e incluíram o número de visitas domiciliares para coleta de leite humano, volume de leite coletado, número de doadoras e de atendimentos individuais realizados no BLH.

\section{Resultados e discussão}

Não foram encontrados na literatura até o momento relato de outros projetos de extensão semelhantes ao apresentado. Comparando dados de 2014, período anterior ao início do projeto, e 2015 correspondente ao período de fevereiro a dezembro, houve um aumento de 104,2\% no número de coletas domiciliares, de 4,6\% no volume de leite humano coletado, de 19,7\% no número de doadoras e de $26,3 \%$ no número de mães atendidas pela equipe do BLH. Os dados de 2016 comparados aos de 2015 demonstraram aumento de 9,2\% no número de visitas domiciliares, de 16,2\% no volume de leite coletado, de 2,8\% no número de doadoras e de 17,8\% nos atendimentos individuais (Quadro 1).

O número de visitas domiciliares, volume de leite coletado, número de doadoras e de atendimentos individuais aumentaram entre os períodos avaliados. Consequentemente, aumentou-se o estoque de leite para o BLH e o número de mulheres apoiadas no processo da amamentação, indicando também o rendimento positivo da continuidade do projeto de extensão. 
Quadro 1 - Parâmetros do Banco de Leite Humano do Hospital São Sebastião avaliados no período de 2014-2016, Viçosa/MG.

\begin{tabular}{lccc}
\hline \multicolumn{1}{c}{ Parâmetros } & \multicolumn{2}{c}{ Ano } \\
\cline { 2 - 4 } & $2014^{*}$ & 2015 & 2016 \\
\hline Visita Domiciliar & 144 & 294 & 321 \\
Volume de Leite coletado (litros) & 168 & 175,8 & 204,3 \\
Número de doadoras & 178 & 213 & 219 \\
Atendimento individual & 1455 & 1838 & 2165 \\
\hline
\end{tabular}

Fonte: FIOCRUZ, 2018 e BLH/Hospital São Sebastião (dados de dezembro de 2016). * Período anterior ao projeto de extensão.

É importante destacar que uma parte do leite coletado acaba sendo descartado após o processo de pasteurização e de análise microbiológica (dados não apresentados), por não atender aos parâmetros de segurança sanitária. A pasteurização visa eliminar agentes patógenos relevantes no leite e, ainda que afete parcialmente as propriedades nutricionais e imunológicas, o leite pasteurizado mantém efeitos benéficos e protetores (BERTINO et al, 2012). A partir desta constatação, a atividade específica do projeto de extensão de orientação à doadora sobre os cuidados higiênicos-sanitários no processo de ordenha e de armazenamento do leite (lavagem das mãos com água e sabão, lavagem das mamas com água, uso de máscara e touca) são de grande importância para diminuir a quantidade de leite descartado.

As nutrizes que pretendem ser doadoras apresentam, muitas vezes, limitação para se deslocarem até o BLH para doarem seu leite, que compreendem desconfortos no pós-parto, atividades domésticas e de cuidado de outros filhos. Dessa forma, o projeto contribui na resolução desse problema uma vez que as doações são coletadas diretamente em domicílio, sendo cômodo para as doadoras, além da facilidade que as mesmas têm de esclarecer suas dúvidas (NEVES, 2011). Além disso, a ordenha de leite humano em domicílio mostrou-se tão segura quanto a ordenha no BLH, desde que sejam seguidas as normas de higiene, conservação, armazenamento e transporte estabelecidas conforme as recomendações da Agência Nacional de Vigilância Sanitária e da Rede Brasileira de BLH (BORGES et al, 2018).

Adicionalmente às coletas domiciliares, atribui-se o aumento de doadoras de leite às campanhas em prol do aleitamento materno desenvolvidas com a comunidade em geral. Estas atividades são de grande importância uma vez que contribuíram para uma maior conscientização da população sobre os benefícios da amamentação e sobre a importância da doação de leite humano, bem como para uma maior divulgação do BLH.

A proporção de prematuridade no Brasil é de 11,3\%, sendo este dado preocupante uma vez que esta condição é considerada um dos principais fatores relacionados à mortalidade infantil (FRANÇA et al, 2017). Entre as causas da mortalidade precoce destaca-se a imaturidade gastrointestinal apresentada pelos prematuros (SUCENA e FURLAN, 2008). Deste modo, tendo em vista que o leite humano apresenta propriedades imunológicas para a estimulação do amadurecimento da defesa e do trofismo do trato gastrointestinal, a amamentação e a doação de leite humano se configuram como importantes estratégias de saúde pública para promoção da saúde e prevenção do óbito infantil (NASCIMENTO e ISSLER, 2004). Estima-se que a ampliação da amamentação a um nível quase universal possa prevenir 823.000 mortes de crianças a cada ano (VICTORA et al, 2016).

Ressalta-se que toda mulher que apresente excesso de leite, seja saudável, não utilize medicamentos que impeçam a doação e se disponha a ordenhar e doar seu leite pode ser uma doadora (BRASIL, 2006). Estudos tem evidenciado que os principais fatores facilitadores para a doação são os benefícios à saúde da criança receptora, o sentimento de solidariedade, o apoio familiar e a coleta domiciliar do leite doado (ABREU et al, 2017; MIRANDA et al, 2017). Entre os principais fatores que dificultam o processo na perspectiva das doadoras são mencionados a falta de tempo para a ordenha (MIRANDA et al, 2017) e o fato de machucar as mamas (ABREU et al, 2017). Estudo de Meneses; Oliveira; Boccolini (2017) realizado em Unidades Básicas de Saúde com posto de recebimento de leite humano mostrou que a ajuda oferecida à nutriz para amamentar se associou a uma prática duas vezes superior de doação de leite materno, ou seja, o apoio ao aleitamento pode produzir outros desdobramentos como a doação do leite. Sendo assim, as ações de saúde e o envolvimento dos profissionais de saúde em prol do aleitamento materno e doação de leite humano se fazem necessárias (RECHIA, 2016). 
Em relação à presença do $B L H$, estudo italiano demonstrou taxas de amamentação na alta hospitalar significativamente maiores nas UTINs com um BLH quando comparado aquelas sem o banco $(29,6 \%$ vs $16 \%$, p<0,05) (BERTINO et al, 2012). Ressalta-se então que a importância dos BLH vai além das funções de coletar, processar e armazenar doações de leite, constituindo-se em um instrumento de grande relevância na promoção e suporte da amamentação (HALLEUX et al, 2017). Nessa perspectiva, o apoio governamental e político por meio de investimentos em recursos humanos e construção de redes de apoio na comunidade pode repercutir nas atividades dos BLH e aumentar as taxas de aleitamento materno e atender a crescente demanda por leite doado (DeMARCHIS et al, 2017).

Em relação ao papel do projeto de extensão na formação do estudante, destaca-se que a vivência de situações externas ao ambiente do campus permite a troca de conhecimentos com a comunidade, o envolvimento com questões sociais e o desenvolvimento de ações de promoção da saúde por meio de um prisma holístico, respeitando as diferentes visões de mundo existentes (SIQUEIRA, et al. 2017). Conforme demonstrado por Almeida; Pereira; Oliveira (2016), o projeto de extensão universitária tem tido papel fundamental ao mostrar a importância das ações extensionistas para uma formação acadêmica mais contextualizada, atendendo às demandas da população.

\section{Conclusões}

As ações extensionistas fortaleceram as atividades do Banco de Leite Humano, aumentando o número de visitas domiciliares, o volume de leite coletado, o número de doadoras e de atendimentos individuais a cada ano, indicando o rendimento positivo da continuidade do projeto de extensão.

Desta forma, verifica-se a que a Extensão Universitária contribui para potencializar ações de saúde pública na promoção e apoio ao aleitamento materno e à doação do leite humano.

\section{Agradecimentos}

Ao Programa Institucional de Bolsas de Extensão Universitária (PIBEX) da Universidade Federal de Viçosa e ao Banco de Leite Humano do Hospital São Sebastião em Viçosa, Minas Gerais.

\section{Referências}

ABREU, J.N. et al. Doação de leite materno: fatores que contribuem com esta prática. Arquivos de Ciências da Saúde, v.24, n.2, p.14-18, 2017.

ALMEIDA, L. E.; PEREIRA, M. N.; OLIVEIRA, V. Governador Valadares (MG) em Extensão: Interfaces para a Dinamização e Instrumentalização do Cenário Extensionista em um Campus Recém-Implantado. Revista Brasileira de Educação Médica, v.40, n.4, p.743-750, 2016.

BERTINO, E. et al. Donor human milk in preterm infant feeding. The Journal of Maternal-Fetal $\mathcal{E}$ Neonatal Medicine, v.25, n.3, 2012.

BERTINO, E. et al. Benefits of donor milk in the feeding of preterm infants. Early Human Development, v. 89 p.S3-S6, 2013.

BORGES, M. S. et al. Quality of human milk expressed in a human milk bank and at home. The Journal of Pediatrics, v. 94, n.4, p.337-454, 2018.

BRASIL. Agência Nacional de Vigilância Sanitária. Resolução-RDC No 171. Dispõe sobre o Regulamento Técnico para o funcionamento de Bancos de Leite Humano. Brasília: Anvisa, 2006.

BRASIL. Agência Nacional de Vigilância Sanitária. Banco de leite humano: funcionamento, prevenção e controle de riscos. Brasília: Anvisa, 2008.

BRASIL. Ministério da Saúde. Secretaria de Atenção à Saúde. Departamento de Atenção Básica. Saúde da criança: Nutrição infantil: aleitamento materno e alimentação complementar. Cadernos de Atenção Básica, n²3. 2. ed. Brasília, 2015. 
CASA DE CARIDADE DE VIÇOSA. Hospital São Sebastião. Fluxos e rotinas - Banco de Leite Humano. Viçosa, MG, 2013.

CORINTIO, M. N. Manual de aleitamento materno. $3^{\text {a }}$ ed. São Paulo: Federação Brasileira das Associações de Ginecologia e Obstetrícia (FEBRASGO), 2015.

DEMARCHIS, A. et al. Establishing an integrated human milk banking approach to strengthen newborn care. Journal of Perinatology, v.37, p.469-474, 2017.

FIOCRUZ. Fundação Oswaldo Cruz. Rede Global de Bancos de Leite Humano. Disponível em: https:/ /rblh.fiocruz.br/pt-br/banco-de-leite-humano-do-hospital-sao-sebastiao. Acesso em: 10 de Outubro de 2017.

FRANÇA, E.B. et al. Principais causas da mortalidade na infância no Brasil, em 1990 e 2015: estimativas do estudo de Carga Global de Doença. Revista Brasileira de Epidemiologia, v.20, n.1, p.4660, 2017.

HALLEUX, V. et al. Use of donor milk in the neonatal intensive care unit. Seminars in Fetal $\mathcal{E}$ Neonatal Medicine, v. 22, n.1, p.23-29, 2017.

ITALIAN ASSOCIATION OF HUMAN MILK BANKS, ARSLANOGLU, S.; BERTINO, E.; TONETTO, P.; DE NISI, G.; AMBRUZZI, A.M.; et al. Guidelines for the establishment and operation of a donor human milk bank. The Journal of Maternal-Fetal $\mathcal{E}$ Neonatal Medicine, v.23 (Suppl. 2), p.120, 2010.

MENESES, T. M. X.; OLIVEIRA, M. I. C.; BOCCOLINI, C. S. Prevalence and factors associated with breast milk donation in banks that receive human milk in primary health care units. The Jornal of Pediatrics, v. 93, n.4, p.382-388, 2017.

MIRANDA, J.O.A. et al. Doação de leite humano: investigação de fatores sociodemográficos e comportamentais de mulheres doadoras. Revista da Associação Brasileira de Alimentação e Nutrição, v.8, n. 1, p.10-17, 2017.

NASCIMENTO, M.B.R.; ISSLER, H. Aleitamento materno em prematuros: manejo clínico hospitalar. Jornal de Pediatria, v. 80, n.5, p.163-172, 2004.

NEVES, L.S. et al. Doação de leite humano: dificuldades e fatores limitantes. O Mundo da Saúde, v.35, n. 2, p. 156-161, 2011.

PEREIRA, M.C.R. et al. O significado da realização da auto-ordenha do leite para as mães dos recém-nascidos prematuros. Revista Gaúcha de Enfermagem, v. 39, p.10-26, 2018.

PRITTAS, T. M.; DRI, C. F. O diálogo entre saúde e política externa na cooperação brasileira em bancos de leite humano. Ciência E Saúde Coletiva, v.22, n.7, p.2277 - 2286, 2017.

QUIGLEY, M.; EMBLETON, N.D.; MCGUIRE, W. Formula versus donor breast milk for feeding preterm or low birth weight infants. Cochrane Database of Systematic Reviews, n. 6, p.1-94, 2018.

RECHIA, F.P.N.S. et al. Fatores que interferem na doação de leite humano: revisão integrativa. Revista Cogitare Enfermagem, v.21, n. 3, p.1-11, 2016.

SIQUEIRA, S. M. C. et al. Atividades extensionistas, promoção da saúde e desenvolvimento sustentável: experiência de um grupo de pesquisa em enfermagem. Escola Anna Nery Revista de Enfermagem, v.21, n.1, p.1-7, 2017.

SUCENA, P.L.; FURLAN, M.F.M.F. Incidência da utilização de leite materno ordenhado em uma Unidade de Terapia Intensiva neonatal e caracterização dos recém-nascidos. Arquivos de Ciências da Saúde, v. 15, n.2, p. 82-9, 2008.

TOSH, K. Feeding preterm infants with formula rather than donor breast milk is associated with faster rates of short term growth, but increased risk of developing necrotising enterocolitis. EvidenceBased Nursing, v.22, n.1, p.18, 2019. 
VICTORA, C.G. et al. Amamentação no século 21: epidemiologia, mecanismos, e efeitos ao longo da vida. Epidemiologia e Serviços de Saúde, v.25, n.1, p.1-24, 2016.

WHO. World Health Organization. Global strategy for infant and young child feeding. WHO; UNICEF. 30p. 2003.

Data de submissão: 18/3/2019. Data de aceite: 22/4/2019. 